\title{
The Energy and Sombor Index of Graphs
}

\author{
Alper Ülker ${ }^{1}$, Arif Gürsoy ${ }^{2}$, Necla Kırcalı Gürsoy ${ }^{3, *}$ \\ ${ }^{1}$ Department of Mathematics, Ağrı İbrahim Çeçen University, \\ 04100, A ğrı, Turkey \\ aulker@agri.edu.tr \\ ${ }^{2}$ Department of Mathematics, Ege University, \\ 35040, Izmir, Turkey \\ arif.gursoy@ege.edu.tr \\ ${ }^{3}$ Tire Kutsan Vocational School, Ege University, \\ 35900, Izmir, Turkey \\ necla.kircali.gursoy@ege.edu.tr
}

(Received July 24, 2021)

\begin{abstract}
The energy of a graph is defined to be the sum of absolute values of eigenvalues of its adjacency matrix. The Sombor index of a graph is a novel topological index of a graph. In this paper, we study relations between energy and Sombor index of a graph $G$ in terms of its degrees. Moreover, we give upper bound for a regular graph in terms of its Sombor index.
\end{abstract}

\section{Introduction}

The graph energy and topological index of a graph are very attractive due to their importance in mathematical chemistry. The graph energy was introduced by Gutman in 1978 [5]. After that, graph energy is continued to be researched by Gutman [6], Zhou et al. [16], and Li et al. [11]. Also, the energy of a vertex is developed by Arizmendi et al. [1]. Moreover, many researchers examine the bounds for graph energy in $[8,10,15,17]$ and also, the energy of the regular graphs are analyzed in $[7,12,14]$.

Recently, a new topological index which is called Sombor index is introduced by Gutman [9] in the chemical graph theory. At the same time, the Sombor index of graphs is a popular work area and newly has been studied in $[2,3,13]$.

\footnotetext{
*Corresponding author.
} 
This paper is concerning with graph energy and Sombor index. In Section 2, we give some fundamental definitions and theorems which will be useful for the rest of the paper. In Section 3, bounds for graph energy are given in terms of its Sombor index. Finally, relation between Sombor index and graph energy is studied for some graph classes in Section 4.

\section{Preliminaries}

Let $G$ be a simple undirected graph without isolated vertices. Let $V(G)$ and $E(G)$ denote the set of vertices and set of edges of $G$, respectively. For any vertex $x \in V(G)$, the degree of a vertex $x$ is denoted by $d_{x}$ which is the number of vertices adjacent to $x$. The maximum degree of a graph is the largest degree among the vertices of $G$ and denoted by $\Delta(G)$. The minimum degree of a graph is the smallest degree among the vertices of a graph and denoted by $\delta(G)$.

Let $G$ be a graph and let $A=A(G) \in M_{n}(\mathbb{M})$ be its adjacency matrix. The energy of the graph $G$ is given by

$$
\varepsilon(G)=\sum_{i=0}^{n}\left|\lambda_{i}\right|,
$$

where $\lambda_{i}$ 's are the eigenvalues of the adjacency matrix of $G$.

The trace of a matrix $M$ is denoted by $\operatorname{Tr}(M)$ and its absolute value $\left(M M^{*}\right)^{1 / 2}$ is denoted by $|M|$. So energy of $G$ is given by

$$
\varepsilon(G)=\operatorname{Tr}(|A(G)|)=\sum_{i=1}^{n}\left(|A(G)|_{i i}\right) .
$$

Next we give the energy of a vertex introduced in [1].

Definition 2.1. The energy of a vertex $x_{i} \in G$ is denoted by $\varepsilon_{G}\left(x_{i}\right)$ and defined by,

$$
\varepsilon_{G}\left(x_{i}\right)=|A(G)|_{i i}, \text { for all } i \in\{1,2, \ldots, n\},
$$

where $|A|=\left(A A^{*}\right)^{1 / 2}$ and $A(G)$ is the adjacency matrix of the graph $G$.

Let $G=(V(G), E(G))$ with $V(G)=\left\{x_{1}, \ldots, x_{n}\right\}$. The energy of a graph is the sum of its vertex energies,

$$
\varepsilon(G)=\varepsilon_{G}\left(x_{1}\right)+\cdots+\varepsilon_{G}\left(x_{n}\right),
$$

so that one can say that the energy of a vertex is a refinement of the its graph. 
Let $e=x y \in E(G)$ for a graph $G$. Then from [4], one can decompose the energy of an edge as,

$$
\varepsilon(e)=\frac{\varepsilon(x)}{d_{x}}+\frac{\varepsilon(x)}{d_{x}}
$$

so that energy of a graph is

$$
\varepsilon(G)=\sum_{e \in E(G)} \varepsilon(e)=\sum_{e=x y \in E(G)}\left(\frac{\varepsilon(x)}{d_{x}}+\frac{\varepsilon(x)}{d_{x}}\right) .
$$

The next theorem gives that the energy of a graph is bounded by square root of the degree of its any vertex.

Theorem 2.2. [1] Let $G$ be a graph and $x \in G$ be any vertex. Then we have $\varepsilon_{G}(x) \leq \sqrt{d_{x}}$. The equality holds if and only if $G \cong K_{1, n}$ where $x$ is the center vertex.

Theorem 2.3. [1] Let $G=(V(G), E(G))$ be a graph with at least one edge. Then for all $x_{i} \in V(G)$,

$$
\varepsilon_{G}\left(x_{i}\right) \geq \frac{d_{i}}{\Delta(G)} .
$$

Equality holds if and only if $G \cong K_{d, d}$.

If $G=(V(G), E(G))$ is a graph with $u, v \in V(G)$, then its Sombor index is

$$
\mathrm{SO}(G)=\sum_{u v \in E(G)} \sqrt{u^{2}+v^{2}}
$$

where $d_{u}$ and $d_{v}$ are degrees of vertices $u$ and $v$, respectively.

\section{Energy and Sombor index}

This section has devoted to the bound results of graph energy in term of its Sombor index.

Theorem 3.1. Let $G$ be a graph with maximum degree $\Delta(G)$. If $\varepsilon(G)$ is its graph energy and $\mathrm{SO}(G)$ is its Sombor index, then $\varepsilon(G) \Delta(G)^{3} \geq \mathrm{SO}(G)$.

Proof. Let $G$ be a graph with vertex set $V(G)$ and edge set $E(G)$. Let $e=x y \in E(G)$ for $x, y \in V(G)$. Since $\varepsilon(e)=\frac{\varepsilon(x)}{d_{x}}+\frac{\varepsilon(y)}{d_{y}}$, by Equations (2.1) and (2.2) then we get,

$$
\varepsilon(G)=\sum_{e \in E} \varepsilon(e)=\sum_{x y \in E(G)}\left(\frac{\varepsilon(x)}{d_{x}}+\frac{\varepsilon(y)}{d_{y}}\right) .
$$


It follows that,

$$
\begin{aligned}
\sum_{e \in E(G)} \varepsilon(e) & =\sum_{x y \in E(G)}\left(\frac{\varepsilon(x)}{d_{x}}+\frac{\varepsilon(y)}{d_{y}}\right) \geq \sum_{x y \in E(G)}\left(\frac{\varepsilon(x)}{d_{x}^{2}}+\frac{\varepsilon(y)}{d_{y}^{2}}\right) \\
& =\sum_{x y \in E(G)}\left(\frac{\varepsilon(x) d_{y}^{2}+\varepsilon(y) d_{x}^{2}}{d_{x}^{2} d_{y}^{2}}\right) .
\end{aligned}
$$

By Theorem 2.3, we have

$$
\begin{aligned}
\sum_{x y \in E(G)}\left(\frac{\varepsilon(x) d_{y}^{2}+\varepsilon(y) d_{x}^{2}}{d_{x}^{2} d_{y}^{2}}\right) & \geq \sum_{x y \in E(G)}\left(\frac{\frac{d_{x}}{\Delta(G)} d_{y}^{2}+\frac{d_{y}}{\Delta(G)} d_{x}^{2}}{d_{x}^{2} d_{y}^{2}}\right) \\
& \geq \sum_{x y \in E(G)}\left(\frac{\sqrt{\frac{d_{x}}{\Delta(G)} d_{y}^{2}+\frac{d_{y}}{\Delta(G)} d_{x}^{2}}}{d_{x} d_{y}}\right) \\
& \geq \sum_{x y \in E(G)}\left(\frac{\sqrt{d_{x}^{2}+d_{y}^{2}}}{\Delta(G)^{3}}\right) .
\end{aligned}
$$

Therefore we conclude that, $\varepsilon(G) \Delta(G)^{3} \geq \mathrm{SO}(G)$.

When $G$ is a regular graph, one can obtain tight bounds in terms of degree.

Theorem 3.2. Let $G$ be a $\Delta$-regular graph. If $\varepsilon(G)$ is its graph energy and $\mathrm{SO}(G)$ is its Sombor index, then $\varepsilon(G) \Delta(G)^{2} \geq \operatorname{SO}(G)$.

Proof. Let $G=(V(G), E(G))$ be a graph. Let $e=x y \in E(G)$ for $x, y \in V(G)$. Since $\varepsilon(e)=\frac{\varepsilon(x)}{d_{x}}+\frac{\varepsilon(y)}{d_{y}}$ by Equation (2.1), then we obtain that,

$$
\begin{aligned}
\varepsilon(G) & =\sum_{x y \in E(G)}\left(\frac{\varepsilon(x)}{d_{x}}+\frac{\varepsilon(y)}{d_{y}}\right) \geq \sum_{x y \in E(G)}\left(\frac{\varepsilon(x)}{d_{x}^{2}}+\frac{\varepsilon(y)}{d_{y}^{2}}\right) \\
& =\sum_{x y \in E(G)}\left(\frac{\varepsilon(x) d_{y}^{2}+\varepsilon(y) d_{x}^{2}}{d_{x}^{2} d_{y}^{2}}\right) .
\end{aligned}
$$

Since $G$ is a regular graph, then $\varepsilon_{G}(x) \geq 1$ for all $x \in V(G)$ by Theorem 2.3. It follows that we can obtain

$$
\sum_{x y \in E(G)}\left(\frac{\varepsilon(x) d_{y}^{2}+\varepsilon(y) d_{x}^{2}}{d_{x}^{2} d_{y}^{2}}\right) \geq \sum_{x y \in E(G)}\left(\frac{d_{x}^{2}+d_{y}^{2}}{d_{x}^{2} d_{y}^{2}}\right)
$$

Then we get

$$
\sum_{x y \in E(G)}\left(\frac{d_{x}^{2}+d_{y}^{2}}{d_{x}^{2} d_{y}^{2}}\right) \geq \sum_{x y \in E(G)}\left(\frac{\sqrt{d_{x}^{2}+d_{y}^{2}}}{d_{x} d_{y}}\right) .
$$


It follows that,

$$
\sum_{x y \in E(G)}\left(\frac{\sqrt{d_{x}^{2}+d_{y}^{2}}}{d_{x} d_{y}}\right) \geq \sum_{x y \in E(G)}\left(\frac{\sqrt{d_{x}^{2}+d_{y}^{2}}}{\Delta(G)^{2}}\right) .
$$

Therefore, $\varepsilon(G) \Delta(G)^{2} \geq \operatorname{SO}(G)$.

Next we give a lemma which will be used in the next theorem.

Lemma 3.3. Let $m$ and $n$ be real numbers. If $m, n>1$, then

$$
m^{2}+n^{2}<m n \sqrt{m^{2}+n^{2}}
$$

Proof. Note that $\sqrt{2 m n} \leq \sqrt{m^{2}+n^{2}}$, whenever $m, n>1$. This leads to $\sqrt{2 m n} \leq$ $\sqrt{m^{2}+n^{2}}<\sqrt{m^{2} n^{2}}$. It follows that $\sqrt{m^{2}+n^{2}}<m n$. This completes the proof.

Theorem 3.4. Let $G$ be a graph with $\delta(G) \geq 2$. Then we have $\varepsilon(G) \leq \operatorname{SO}(G)$.

Proof. Note that $\varepsilon(G)=\sum_{x y \in E(G)}\left(\frac{\varepsilon(x)}{d_{x}}+\frac{\varepsilon(y)}{d_{y}}\right)$. From this we get,

$$
\varepsilon(G)=\sum_{x y \in E(G)}\left(\frac{\varepsilon(x)}{d_{x}}+\frac{\varepsilon(y)}{d_{y}}\right)=\sum_{x y \in E(G)}\left(\frac{\varepsilon(x) d_{y}+\varepsilon(y) d_{x}}{d_{x} d_{y}}\right) .
$$

Then by Theorem 2.2, we can deduce that

$$
\sum_{x y \in E(G)}\left(\frac{\varepsilon(x) d_{y}+\varepsilon(y) d_{x}}{d_{x} d_{y}}\right) \leq \sum_{x y \in E(G)}\left(\frac{d_{x} d_{y}+d_{y} d_{x}}{d_{x} d_{y}}\right),
$$

since $\varepsilon_{G}(x) \leq d_{x}$ and $\varepsilon_{G}(y) \leq d_{y}$.

The inequality for real numbers $d_{x}^{2}+d_{y}^{2} \geq 2 d_{x} d_{y}$ from algebra implies that

$$
\sum_{x y \in E(G)}\left(\frac{d_{x} d_{y}+d_{y} d_{x}}{d_{x} d_{y}}\right) \leq \sum_{x y \in E(G)}\left(\frac{d_{x}^{2}+d_{y}^{2}}{d_{x} d_{y}}\right) .
$$

Then by Lemma 3.3, we get

$$
\sum_{x y \in E(G)}\left(\frac{d_{x}^{2}+d_{y}^{2}}{d_{x} d_{y}}\right) \leq \sum_{x y \in E(G)} \sqrt{d_{x}^{2}+d_{y}^{2}}=\operatorname{SO}(G)
$$

since $d_{x}, d_{y} \geq 2$ by assumption $\delta(G) \geq 2$.

Theorem 3.5. Let $G$ be a regular graph. Then $\varepsilon(G) \leq \operatorname{SO}(G)^{2}$. The equality holds if $G \cong K_{2}$. 
Proof. The energy of a graph can be calculated as:

$$
\varepsilon(G)=\sum_{e \in E(G)} \varepsilon(e)=\sum_{e=x y \in E(G)}\left(\frac{\varepsilon(x)}{d_{x}}+\frac{\varepsilon(y)}{d_{y}}\right) .
$$

Then we get

$$
\sum_{x y \in E(G)}\left(\frac{\varepsilon(x)}{d_{x}}+\frac{\varepsilon(y)}{d_{y}}\right)=\sum_{x y \in E(G)}\left(\frac{\varepsilon(x) d_{y}+\varepsilon(y) d_{x}}{d_{x} d_{y}}\right) .
$$

Since $G$ is regular, it follows that,

$$
\begin{aligned}
\sum_{x y \in E(G)}\left(\frac{\varepsilon(x) d_{y}+\varepsilon(y) d_{x}}{d_{x} d_{y}}\right) & \leq \sum_{x y \in E(G)}\left(\frac{d_{x}^{2}+d_{y}^{2}}{d_{x} d_{y}}\right) \\
& \leq \sum_{x y \in E(G)}\left(d_{x}^{2}+d_{y}^{2}\right) \\
& =\operatorname{SO}(G)^{2}
\end{aligned}
$$

by Theorem 2.2. Also if $G \cong K_{2}$, then by Theorem 2.2 and 2.3 , one can easily conclude that $\varepsilon(G)=\mathrm{SO}(G)^{2}$.

\section{Energy and Sombor index relations of graph classes}

In this section we give some relations of graph energy and Sombor index for some graph classes.

Theorem 4.1. Let $G$ be a complete graph with $n$ vertices. Then

$$
\mathrm{SO}(G)=\frac{n(n-1)}{2 \sqrt{2}} \varepsilon(G)
$$

Proof. Assume that $G$ is a complete graph with $n$ vertices. Then it is clear that $\varepsilon(G)=$ $2(n-1)$. Since $d_{x}=(n-1)$ for any vertex $x \in K_{n}$, then Sombor index of $K_{n}$ is

$$
\begin{aligned}
\mathrm{SO} & =\sum_{e=x y \in E(G)} \sqrt{d_{x}^{2}+d_{y}^{2}} \\
& =\sum_{e \in E(G)} \sqrt{2(n-1)^{2}} \\
& =\left(\begin{array}{l}
n \\
2
\end{array}\right)(n-1) \sqrt{2} .
\end{aligned}
$$

Therefore, we get

$$
\mathrm{SO}(G)=\frac{n(n-1)}{2 \sqrt{2}} \varepsilon(G)
$$


Theorem 4.2. Let $G$ be a complete bipartite graph $K_{n, n}$. Then

$$
\frac{\sqrt{2}}{8} \varepsilon(G)^{3}=\operatorname{SO}(G) .
$$

Proof. Since $G=K_{n, n}$, then combining Theorem 2.2 and Theorem 2.3 implies that $\varepsilon_{G}(x)=1$, for any vertex $x \in K_{n, n}$. It follows that $\varepsilon\left(K_{n, n}\right)=2 n$. Also,

$$
\operatorname{SO}\left(K_{n, n}\right)=\sum_{x y \in E\left(K_{n, n}\right)}\left(\sqrt{2 n^{2}}\right)=n^{3} \sqrt{2} .
$$

Combining the arguments leads to

$$
\frac{\sqrt{2}}{8} \varepsilon\left(K_{n, n}\right)^{3}=\operatorname{SO}\left(K_{n, n}\right) .
$$

\section{References}

[1] O. Arizmendi, J. F. Hidalgo, O. Juarez-Romero, Energy of a vertex, Lin. Algebra Appl. 557 (2018) 464-495.

[2] R. Cruz, I. Gutman, J. Rada, Sombor index of chemical graphs, Appl. Math. Comput. 399 (2021) \#126018.

[3] K. C. Das, A. S. Çevik, I. N. Cangul, Y. Shang, On Sombor index, Symmetry 13 (2021) \#140.

[4] T. Došlić, T. Réti, D. Vukičević, On the vertex degree indices of connected graphs, Chem. Phys. Lett. 512 (2011) 283-286.

[5] I. Gutman, The energy of a graph, Ber. Math. Stat. Sekt. Forschungsz. Graz 103 (1978) 1-22.

[6] I. Gutman, The energy of a graph: old and new results, in: A. Betten, A. Kohnert, R. Laue, A. Wassermann (Eds.), Algebraic Combinatorics and Applications, Springer, Berlin, 2001, pp. 196-211.

[7] I. Gutman, S. Z. Firoozabadi, J. A. de la Peña, J. Rada, On the energy of regular graphs, MATCH Commun. Math. Comput. Chem. 57 (2007) 435-442.

[8] I. Gutman, M. R. Oboudi, Bounds on graph energy, Discr. Math. Lett. 4 (2020) 1-4.

[9] I. Gutman, Geometric approach to degree-based topological indices: Sombor indices, MATCH Commun. Math. Comput. Chem. 86 (2021) 11-16. 
[10] A. Jahanbani, Lower bounds for the energy of graphs, AKCE Int. J. Graphs Comb. 15 (2018) 88-96.

[11] X. Li, Y. Shi, I. Gutman, Graph Energy, Springer, New York, 2012.

[12] V. Nikiforov, Remarks on the energy of regular graphs, Lin. Algebra Appl. 508 (2016) $133-145$.

[13] T. Réti, T. Došlić, A. Ali, On the Sombor index of graphs, Contrib. Math. 3 (2021) $11-18$.

[14] E. R. van Dam, W. H. Haemers, J. H. Koolen, Regular graphs with maximal energy per vertex, J. Comb. Theory B 107 (2014) 123-131.

[15] L. Wang, X. Ma, Bounds of graph energy in terms of vertex cover number, Lin. Algebra Appl. 517 (2017) 207-216.

[16] B. Zhou, I. Gutman, J. A. de la Peña, J. Rada, L. Mendoza, On spectral moments and energy of graphs, MATCH Commun. Math. Comput. Chem. 57 (2007) 183-191.

[17] Q. Zhou, D. Wong, D. Sun, A lower bound for graph energy, Lin. Multilin. Algebra 68 (2020) 1624-1632. 